\title{
Revisión: Beneficios del ejercicio físico en pacientes con enfermedad renal crónica en hemodiálisis
}

\author{
María José Fernández Lara ${ }^{1,2}$, José Luis Ibarra Cornejo ${ }^{3}$, Elena Viviana Aguas Alveal ${ }^{1}$, Cesar Eduardo González \\ Tapia $^{3}$, Diego Galvarino Quidequeo Reffers ${ }^{3}$ \\ ${ }^{1}$ Licencias en Enfermería con formación en Hemodiálisis. Grupo de investigación Ciencia en Movimiento. Chile \\ ${ }^{2}$ Alumna de Magister en Enfermería. Universidad Católica del Maule. Chile \\ ${ }^{3}$ Licenciados en Kinesiología. Grupo de investigación Ciencia en Movimiento. Chile
}

\section{Resumen}

Introducción: La enfermedad renal crónica (ERC) en etapa terminal requiere de tratamiento de sustitución renal como lo es la hemodiálisis. Los pacientes que se someten a este tipo de tratamiento se caracterizan por tener una alta prevalencia de enfermedades cardiovasculares asociadas 0 exacerbadas por la inactividad física y alteraciones del metabolismo mineral óseo que llevan a presentar también problemas psicológicos y bajos niveles de calidad de vida.

Objetivo: Identificar los beneficios del ejercicio en pacientes con ERC que se someten a hemodiálisis.

Metodología: Se realizó una revisión bibliográfica en las bases de datos Pubmed, Scielo, Google académico y Pedro.

Resultados: Se seleccionaron 25 artículos, donde la información obtenida se organizó de acuerdo a las variables evaluadas en los estudios. Se encontró que el ejercicio en pacientes en HD tiene efectos beneficiosos a nivel cardiovascular, en el metabolismo óseo, en la eliminación de solutos, en la función y capacidad física, a nivel psicológico y en la calidad de vida.

Conclusiones: El ejercicio físico tiene una gran cantidad de beneficios para los pacientes en tratamiento dialítico por lo que incluir programas de ejercicios intradialíticos puede ser una forma económica de ayudar

Correspondencia:
EU María José Fernández Lara
Pje. Los Claretianos 1768, San Vicente de Tagua.Tagua. Chile
E mail: MjfernandezL31@gmail.com

a minimizar problemas asociados a la enfermedad renal crónica, no solo en el área fisiológica del paciente, sino también en el área psicológica y social.

PALABRAS CLAVE: hemodiálisis; ejercicio intradiálisis.

\section{Review: Benefits of physical exercise in patients with chronic kidney disease on hemodialysis}

\section{Abstract}

Introduction: End stage renal disease requires renal replacement therapy, such as haemodialysis. Patients who undergo this type of treatment are characterized by a high prevalence of cardiovascular diseases associated or exacerbated by physical inactivity and alterations of bone mineral metabolism that lead to psychological problems and low levels of quality of life.

Objective: To identify the benefits of exercise in patients with chronic kidney disease on hemodialysis.

Methodology: A bibliographic review was performed in PubMed, Scielo, Google Scholar and Pedro databases.

Results: We selected 25 articles. Information was organized according to the variables evaluated in the studies. It was found that exercise in haemodialysis patients has beneficial effects at cardiovascular level, bone metabolism, solutes removal, physical function and capacity, psychological level and quality of life. 
Conclusions: Physical exercise has a great number of benefits for patients undergoing dialysis treatment. Intra-dialysis exercise programs can be an inexpensive way to help minimize problems associated with chronic kidney disease, not only in the physiological area of the patient, but also in the psychological and social area.

KEYWORDS: hemodialysis; exercise; intradialysis.

\section{Introducción}

La enfermedad renal crónica (ERC) es un problema de salud pública a nivel mundial por su creciente prevalencia e incidencia y las complicaciones que esta conlleva. La etapa terminal de la ERC requiere un tratamiento de sustitución renal como lo es la hemodiálisis y el trasplante renal. En Chile, la tasa de pacientes en estadio $\mathrm{V}$ (terminal) que se encuentran en hemodiálisis (HD) por millón de habitantes a la fecha de agosto de 2015 es de $1.060^{1}$. Estos se caracterizan por una alta prevalencia de enfermedades cardiovasculares asociadas o exacerbadas por la inactividad física, siendo considerado este último como un factor importante de mortalidad ${ }^{2,3}$.

La mayoría de los pacientes que reciben tratamiento de HD lo hacen de manera trisemanal, con una duración de 4 horas por sesión. Estas horas son consideradas un periodo de inactividad forzada que, junto a la edad avanzada, la neuropatía y miopatía urémica, el catabolismo proteico alterado y la anemia, acrecientan la debilidad muscular, disminuyen la capacidad funcional y aumentan la morbi-mortalidad de estos pacientes, provocando también un deterioro a nivel psicológico y una considerable disminución en su calidad de vida ${ }^{2,3,4}$.

Se han publicado varios estudios acerca de programas de ejercicio físico para pacientes renales en HD, como de rehabilitación domiciliaria, de ejercicio aeróbico y de fuerza-resistencia muscular, y la mayoría muestra efectos beneficiosos tanto a nivel fisiológico, como funcional y psicológico ${ }^{2,4}$. En Chile no existen programas estandarizados sobre ejercicio para esta población por lo que se planteó como objetivo determinar los efectos de los diferentes tipos de ejercicio en pacientes con enfermedad renal crónica que se encuentran recibiendo tratamiento de hemodiálisis.

\section{Material y Método}

Se realizó una revisión bibliográfica en las bases de datos Pubmed, Scielo, Google académico y Pedro, con las palabras claves: hemodialysis, exercise, intradialytic.

Se seleccionaron artículos que cumplieran con los siguientes criterios de inclusión:

- Investigaciones primarias y revisiones.

- Artículos publicados desde enero del 2007 hasta enero del 2017.

- Con texto en español, inglés y portugués.

Se encontraron 210 artículos atingentes con el tema a los cuales se les aplicó un filtro con los criterios de búsqueda. Luego se realizó una lectura crítica de los títulos y resúmenes, en donde se seleccionó un total de 25 artículos. Dichos artículos fueron leídos por los cinco investigadores realizando una ficha de extracción de cada uno con la información más relevante. La información obtenida se organizó por la autora principal de acuerdo con las variables evaluadas en los estudios.

\section{Resultados}

Los artículos incluidos en la revisión se resumen en la Tabla 1.

Tabla 1. Resumen de artículos seleccionados.

\begin{tabular}{|c|c|c|c|c|c|}
\hline Título/Año & Autores & $\begin{array}{l}\text { Tipo } \\
\text { de estudio }\end{array}$ & Tamaño muestral & Actividad realizada & Conclusiones principales \\
\hline $\begin{array}{l}\text { Efectos de un programa } \\
\text { de ejercicio de fuer- } \\
\text { za-resistencia muscular } \\
\text { en la capacidad funcio- } \\
\text { nal, fuerza y calidad de } \\
\text { vida de adultos con en- } \\
\text { fermedad renal crónica } \\
\text { en hemodiálisis. } 2016\end{array}$ & $\begin{array}{l}\text { Cigarroa I., } \\
\text { Barriga C., } \\
\text { Michéas C., } \\
\text { Zapata R., } \\
\text { Soto C. }\end{array}$ & $\begin{array}{l}\text { Estudio } \\
\text { longitudinal, } \\
\text { pre-experi- } \\
\text { mental }\end{array}$ & $\begin{array}{l}13 \text { pacientes fueron re- } \\
\text { clutados de una clínica } \\
\text { de diálisis de Los Án- } \\
\text { geles (Chile) que cum- } \\
\text { plieron los criterios de } \\
\text { inclusión }\end{array}$ & $\begin{array}{l}\text { El programa se realizó con } 2 \text { sesio- } \\
\text { nes por semana durante } 8 \text { semanas } \\
\text { en el Laboratorio de Fisiología del } \\
\text { Ejercicio de la Universidad Santo } \\
\text { Tomás (UST), Los Ángeles (Chile). } \\
\text { Cada sesión duró } 40 \text { min y se hizo } \\
1 \text { hora antes de la HD. Las evalua- } \\
\text { ciones y el programa fueron reali- } \\
\text { zados por un kinesiólogo diferente }\end{array}$ & $\begin{array}{l}\text { En conclusión, pacientes con } \\
\text { ERC en HD de al menos un } \\
\text { año, sometidos a un programa } \\
\text { de ejercicios fuerza-resisten- } \\
\text { cia } 2 \text { veces por semana du- } \\
\text { rante } 8 \text { semanas, mejoraron } \\
\text { parámetros de fuerza mus- } \\
\text { cular, capacidad funcional y } \\
\text { calidad de vida relaciona con } \\
\text { la salud }\end{array}$ \\
\hline
\end{tabular}




\begin{tabular}{|l|l|l|l|}
\hline Título/Año & Autores & $\begin{array}{l}\text { Tipo } \\
\text { de estudio }\end{array}$ & Tamaño muestral \\
\hline $\begin{array}{l}\text { Ejercicio en pacientes } \\
\text { en hemodiálisis: revi- } \\
\text { sión sistemática de la } \\
\text { literatura. } 2010\end{array}$ & $\begin{array}{l}\text { Segura Ortí } \\
\text { E. }\end{array}$ & $\begin{array}{l}\text { Revisión } \\
\text { sistemática, } \\
\text { meta-aná- } \\
\text { lisis }\end{array}$ & $\begin{array}{l}\text { Cumplieron los criterios } \\
\text { de inclusión 16 estu- } \\
\text { dios. Una publicación } \\
\text { se acompañó de dos } \\
\text { estudios posteriores que } \\
\text { trataban los mismos te- } \\
\text { mas, por lo que se contó } \\
\text { como un solo estudio } \\
\text { para el análisis (14 es- } \\
\text { tudios en total) }\end{array}$ \\
\hline
\end{tabular}

Vascular effects of exercise training in CKD: current evidence and pathophysiological mechanisms. 2015

Enfermedad cardiovascular y función renal. Mecanismos patogénicos. 2008
Amaryllis Revisión Incluyó 13 estudios los H., Van narrativa cuales fueron categoCraenenbroeck, Emeline M. Van Craenenbroeck

González Maqueda, Casanova Rodríguez rizados en dos grupos Pre-diálisis y hemodiálisis
Selección de estudios con intervención de ejercicios en pacientes con enfermedad renal crónica, de texto completo en inglés

Se seleccionaron ensayos clínicos aleatorios (ECA) que compararon una intervención que incluía un componente de ejercicios con un control (sin ejercicio o con ejercicio de intensidad muy baja equivalente a placebo), una intervención sin ejercicios o una intervención con ejercicios de distinta naturaleza en pacientes en HD

En los 14 estudios revisados se han incluido a un total de 640 pacientes. El tamaño muestral más pequeño incluyó a 13 pacientes, frente al mayor tamaño muestral que incluyó a 103 pacientes. Sólo un estudio incluyó a más de 100 pacientes
Conclusiones principales

El ejercicio aeróbico, aislado o combinado con ejercicio de fuerza, mejora la capacidad de ejercicio. El ejercicio de fuerza mejora la capacidad funcional del sujeto, la fuerza de los miembros inferiores $y$ la calidad de vida

Se recomienda que el ejercicio durante la HD se realice en las primeras 2 horas de tratamiento

Aunque la percepción general es que el ejercicio en pacientes en HD es seguro, se recomienda que futuros estudios señalen de forma detallada cuáles han sido los efectos adversos de la intervención. La realización de una valoración previa del sujeto y la supervisión del programa por parte de un profesional capacitado son los requisitos mínimos para implantar este tipo de programas en las unidades de HD

El entrenamiento con ejercicio aumenta la capacidad de ejercicio y la función física en pacientes con enfermedad renal, y ambos son factores predictivos de mortalidad en esta población

Los pacientes con insuficiencia renal crónica tienen más probabilidad de morir por causa cardiovascular que de progresar a insuficiencia renal terminal. La excreción urinaria de albúmina es un marcador de riesgo tanto de enfermedad renal como de enfermedad cardiovascular en diabéticos y no diabéticos. En la insuficiencia renal crónica, la hipertensión, la dislipemia y la diabetes mellitus son los principales factores de riesgo de disfunción endotelial e inflamación

La potente asociación causal entre ERC y enfermedad CV hace imperativo prevenir el progreso de la ERC, ya que con ello se reduce el riesgo cardiovascular. En los pacien- 


\begin{tabular}{|c|c|c|c|c|c|}
\hline Título/Año & Autores & $\begin{array}{l}\text { Tipo } \\
\text { de estudio }\end{array}$ & Tamaño muestral & Actividad realizada & Conclusiones principales \\
\hline & & & & & $\begin{array}{l}\text { tes con ERC la causa de este } \\
\text { riesgo cardiovascular elevado } \\
\text { es multifactorial, explicada } \\
\text { en parte por procesos fisiopa- } \\
\text { tológicos propios } \\
\text { Se deben explorar estrategias } \\
\text { innovadoras, centradas en los } \\
\text { mecanismos fisiopatológicos } \\
\text { propios de esta población, sin } \\
\text { olvidar que es vital iniciar la } \\
\text { prevención en forma precoz } \\
\text { y enfocar el tratamiento de } \\
\text { estos pacientes de forma mul- } \\
\text { tifactorial y bajo un equipo } \\
\text { multidisciplinario }\end{array}$ \\
\hline $\begin{array}{l}\text { Role of exercise in the } \\
\text { prevention of cardiovas- } \\
\text { cular disease: Results, } \\
\text { mechanisms, and new } \\
\text { perspectives. } 2013\end{array}$ & $\begin{array}{l}\text { Schuler G., } \\
\text { Adams V., } \\
\text { Goto Y. }\end{array}$ & $\begin{array}{l}\text { Revisión } \\
\text { narrativa }\end{array}$ & No especifica & No especifica & $\begin{array}{l}\text { La actividad física es uno de } \\
\text { los factores fundamentales } \\
\text { para mantener la salud y } \\
\text { evitar los factores de ries- } \\
\text { go; el cumplimiento a largo } \\
\text { plazo, sin embargo, es pobre } \\
\text { en la gran mayoría de los } \\
\text { pacientes. Hasta hoy, todas } \\
\text { las estrategias para mejorar } \\
\text { la adherencia han fracasado } \\
\text { significativamente y las ten- } \\
\text { dencias a largo plazo parecen } \\
\text { apuntar en la dirección equi- } \\
\text { vocada }\end{array}$ \\
\hline
\end{tabular}

Benefits of Exercise Training during Hemodialysis Sessions: A Prospective Cohort Study. 2013

\section{Mihaescu \\ A., Avram \\ C., Bob F.}

Estudio prospectivo de cohorte.
Grupo de estudio ( $n=19)$ $y$ el grupo control $(n=16)$ de pacientes con hemodiálisis crónica de Timisoara, Rumania El objetivo de este estudio fue determinar el impacto de 3 meses de entrenamiento físico durante la diálisis sobre algunos de los factores de riesgo cardiovascular

Exercise capacity and circulating endothelial progenitor cells in hemodialysis patients. 2007
Rigolin G., Malagoni A., Catizone

L.

Estudio de cohorte no randomizado
Fueron divididos en dos grupos: En el primer grupo se prescribieron ejercicio físico (16), mientras que al otro grupo (14) no se prescribió ejercicio (control). Se incluyeron pacientes que habían estado en hemodiálisis por $<1$ año, fueron excluidos pacientes con enfermedades agudas o inestables
La intervención: 40 minutos de entrenamiento (con la mano sin fístula y ambas extremidades inferiores) durante cada sesión de hemodiálisis durante 3 meses, se aplicó solo al grupo de estudio. Las mediciones realizadas antes y después de la intervención fueron aórtica velocidad de la onda del pulso (VOP), índice de aumento aórtico, el tiempo y la presión arterial central y periférico para la evaluación de la rigidez arterial de regreso, la composición corporal por bioimpedancia multifrecuencia y el rendimiento físico

El entrenamiento físico durante la diálisis tiene un efecto positivo sobre la rigidez arterial, la composición corporal y el rendimiento físico de los pacientes hemodializados crónicos

Este estudio evaluó la respuesta de células progenitoras endoteliales (EPCs) a un programa de 6 meses de ejercicio de moderada intensidad en pacientes en hemodiálisis
En conclusión, un programa de ejercicio de intensidad moderada aumentó de forma significativa y selectiva el número de EPCs en pacientes en hemodiálisis 


\begin{tabular}{|c|c|c|c|}
\hline Título/Año & Autores & $\begin{array}{l}\text { Tipo } \\
\text { de estudio }\end{array}$ & Tamaño muestral \\
\hline $\begin{array}{l}\text { Exercise training for } \\
\text { adults with chronic } \\
\text { kidney disease. } 2011\end{array}$ & $\begin{array}{l}\text { Heiwe S., } \\
\text { Jacobson S. }\end{array}$ & $\begin{array}{l}\text { Meta-aná- } \\
\text { lisis }\end{array}$ & $\begin{array}{l}45 \text { estudios aleoteri- } \\
\text { zados con un total de } \\
1863 \text { participantes, en } \\
\text { donde solo treinta y dos } \\
\text { estudios presentaron } \\
\text { datos que podrían ser } \\
\text { meta analizados. } \\
\text { Los objetivos de este es- } \\
\text { tudio fueron } 1 \text { ) evaluar } \\
\text { los efectos del ejercicio } \\
\text { regular en adultos con } \\
\text { ERC y pacientes con } \\
\text { trasplante de riñón; y } \\
\text { 2) determinar cómo se } \\
\text { debe diseñar el progra- } \\
\text { ma de ejercicios (por } \\
\text { ejemplo, tipo, duración, } \\
\text { intensidady frecuencia } \\
\text { de ejercicio) }\end{array}$ \\
\hline $\begin{array}{l}\text { Association between } \\
\text { physical performance } \\
\text { and allcause mortality } \\
\text { in CKD. } 2013\end{array}$ & $\begin{array}{l}\text { Roshan- } \\
\text { ravan B., } \\
\text { Cohen R., } \\
\text { Ayers P., } \\
\text { Littman A., } \\
\text { de Boer I. }\end{array}$ & $\begin{array}{l}\text { Análisis de } \\
\text { estudio de } \\
\text { cohorte }\end{array}$ & $\begin{array}{l}\text { Fueron evaluados } 385 \\
\text { pacientes de forma } \\
\text { ambulatoria con un } \\
\text { promedio de edad de } \\
61 \text { que se encontraban } \\
\text { entre las etapas } 2-4 \text { de } \\
\text { la insuficiencia renal. El } \\
\text { objetivo de este estudio } \\
\text { fue evaluar la asocia- } \\
\text { ción entre el rendimien- } \\
\text { to físico y las causas } \\
\text { de muertes en los pa- } \\
\text { cientes con enfermedad } \\
\text { renal }\end{array}$ \\
\hline
\end{tabular}

Marinho S., Estudio exMoraes C., perimental Barbosa J.
Exercise training alters the bone mineral density of hemodialysis patients. 2012

21 pacientes fueron divididos en dos grupos; 10 pacientes en el grupo de entrenamiento físico con 46.9 de edad; y 11 pacientes que integraron el grupo control con un promedio de edad de 50.5 años. Este estudio buscaba medir los efectos de un entrenamiento intradialítico de resistencia física sobre la densidad mineral ósea en pacientes en HD

Valoración física, condición física y calidad de vida en pacientes con diferentes tratamientos renales sustitutivos. 2015

\section{Actividad realizada}

Conclusiones principales

Los tipos de entrenamiento físico incluyeron entrenamiento cardiovascular, entrenamiento mixto cardiovascular y de resistencia, entrenamiento de resistencia solo y yoga. Algunos estudios usaron intervenciones de ejercicios supervisados y otros usaron intervenciones no supervisadas. La intensidad del ejercicio se clasificó como "alta" o "baja", la duración de las sesiones de ejercicio individuales varió de 20 minutos / sesión a 110 minutos / sesión, y la duración del estudio fue de dos a 18 meses

Fue comparado la fuerza de empuñadura, velocidad de marcha usual, test de tiemd up and go, y test de marcha en 6 minutos con sus valores normales y con la asociación de todas las causas de muertes

El grupo de ejercicio realizó entrenamiento con bandas elásticas y con pesos libres de tobillo en ambas extremidades inferiores, 3 veces por semana durante 24 semanas con un total de 72 sesiones

Se utilizó la absorciometría de rayos $X$ de energía dual para medir la densidad ósea

25 enfermos renales crónicos varones de entre 59 y 72 años, que participaron voluntariamente en este estudio, fueron divididos en 3 grupos: trasplantados Renales: $\mathrm{N}=11$, hemodializados: $N=6$, y en tratamiento de diálisis
Se les administró el cuestionario de cuantificación de actividad física YALE, el cuestionario de calidad de vida $K D Q \mathrm{~L}$ y realizaron la batería de tests Senior Fitness Test (SFT). Todas las evaluaciones se llevaron a cabo en horario de tarde y en días interdiálisis para los hemodializados. Cada sujeto asistió al laboratorio un solo día, donde se le aplicó en
En conclusión, Solo en el grupo de ejercicio, el porcentaje de osteoporosis se redujo al $10.0 \%$. Por lo tanto, este estudio sugiere que el ejercicio de resistencia puede ser útil para mejorar la densidad osea en pacientes con HD
Los datos indican que no existen diferencias significativas en niveles de actividad física, calidad de vida y condición física al comparar a sujetos con ERC sometidos a tres tratamientos sustitutivos diferentes. Futuros estudios deberían reforzar esta conclusión mediante el uso de 


\begin{tabular}{|c|c|c|c|c|c|}
\hline Título/Año & Autores & $\begin{array}{l}\text { Tipo } \\
\text { de estudio }\end{array}$ & Tamaño muestral & Actividad realizada & Conclusiones principales \\
\hline & & & $\begin{array}{l}\text { peritoneal: } \mathrm{N}=8 \\
\text { El objetivo de este es- } \\
\text { tudio fue comparar el } \\
\text { nivel de actividad física, } \\
\text { condición física y cali- } \\
\text { dad de vida en enfer- } \\
\text { mos renales crónicos } \\
\text { sometidos a HD, DP o } \\
\text { trasplante }\end{array}$ & $\begin{array}{l}\text { primer lugar el cuestionario de ac- } \\
\text { tividad física YALE, posteriormente } \\
\text { el cuestionario KDQoL y por último } \\
\text { los test físicos }\end{array}$ & $\begin{array}{l}\text { herramientas diferentes y } \\
\text { muestras mayores }\end{array}$ \\
\hline $\begin{array}{l}\text { Efeitos do exercício } \\
\text { aeróbio durante a he- } \\
\text { modiálise em pacientes } \\
\text { com doença renal } \\
\text { crônica: uma revisão da } \\
\text { literatura. } 2012\end{array}$ & $\begin{array}{l}\text { Böhm J., } \\
\text { Borba M., } \\
\text { Saldanha F. }\end{array}$ & $\begin{array}{l}\text { Revisión } \\
\text { Sistemática }\end{array}$ & $\begin{array}{l}\text { El objetivo de este es- } \\
\text { tudio fue describir los } \\
\text { efectos del ejercicio } \\
\text { físico en pacientes con } \\
\text { ERC sometidos a pro- } \\
\text { gramas de ejercicios } \\
\text { físicos aeróbicos en } \\
\text { cicloergómetro para } \\
\text { miembros inferiores du- } \\
\text { rante la HD } \\
\text { Para lograr el objetivo } \\
\text { fueron consultados las } \\
\text { bases de datos de Med- } \\
\text { line, PubMed, Scielo, } \\
\text { Embase e ScienceDi- } \\
\text { rect, entre enero del } \\
2002 \text { y julio del } 2010 \\
\text { idiomas, portugués e } \\
\text { inglés }\end{array}$ & $\begin{array}{l}\text { Para la selección de los artículos } \\
\text { inicialmente fue la lectura de los } \\
\text { resúmenes y títulos verificando } \\
\text { que contengan los criterios de in- } \\
\text { clusión y que evaluaran los efectos } \\
\text { del ejercicio aeróbico realizando } \\
\text { cicloergómetro en miembros infe- } \\
\text { riores en paciente con HD }\end{array}$ & $\begin{array}{l}\text { El estudio concluyó que la } \\
\text { realización de ejercicios ae- } \\
\text { róbicos durante la HD mejora } \\
\text { la capacidad funcional, pro- } \\
\text { porcionando beneficios car- } \\
\text { diorespiratorio y muscular a } \\
\text { los pacientes }\end{array}$ \\
\hline $\begin{array}{l}\text { Beneficial effects of } \\
\text { an intradialytic cycling } \\
\text { training program in } \\
\text { patients with end-stage } \\
\text { kidney disease. } 2015\end{array}$ & $\begin{array}{l}\text { Groussard } \\
\text { C., Rou- } \\
\text { chon-Isnard } \\
\text { M., Coutard } \\
\text { C. }\end{array}$ & $\begin{array}{l}\text { Estudio } \\
\text { clínico ex- } \\
\text { perimental }\end{array}$ & $\begin{array}{l}\text { Dieciocho pacientes } \\
\text { en hemodiálisis fueron } \\
\text { asignados aleatoria- } \\
\text { mente un grupo de en- } \\
\text { trenamiento (8) y en un } \\
\text { grupo de control (10) } \\
\text { El objetivo fue determi- } \\
\text { nar si un protocolo de } \\
\text { entrenamiento aeróbico } \\
\text { intradiálisis en cicloer- } \\
\text { gometro, podría reducir } \\
\text { el estrés oxidativo y } \\
\text { mejorar otros trastor- } \\
\text { nos relacionados con la } \\
\text { ERC, como la composi- } \\
\text { ción corporal alterada y } \\
\text { el perfil lipídico }\end{array}$ & $\begin{array}{l}\text { El grupo de ejercicio intradiálisis } \\
\text { realizó ciclismo: } 30 \text { minutos, } 55 \% \\
-60 \% \text { de potencia máxima, } 3 \text { días / } \\
\text { semana, por } 3 \text { meses } \\
\text { Fueron evaluados la Composición } \\
\text { corporal, condición física (consumo } \\
\text { máximo de oxígeno y prueba de } \\
\text { caminata de } 6 \text { minutos (6MWT)), } \\
\text { perfil de lípidos (triglicéridos (TG), } \\
\text { colesterol total, lipoproteína de } \\
\text { alta densidad y baja lipoproteína } \\
\text { de densidad (LDL)) y estado pro / } \\
\text { antioxidante (L-F2œ-isoprostanos } \\
\text { (F2-IsoP) y LDL oxidada en plas- } \\
\text { ma }\end{array}$ & $\begin{array}{l}\text { Los resultados mostraron } \\
\text { que } 30 \text { minutos de entrena- } \\
\text { miento intradialítico, } 3 \text { veces } \\
\text { por semana durante } 3 \text { meses, } \\
\text { son suficientes para ejercer } \\
\text { efectos beneficiosos sobre } \\
\text { el marcador más sensible y } \\
\text { confiable de la peroxidación } \\
\text { lipídica (IsoP) al tiempo que } \\
\text { mejora los trastornos asocia- } \\
\text { dos a la ERC (perfil lipídico y } \\
\text { estado físico) }\end{array}$ \\
\hline $\begin{array}{l}\text { Exercise training in } \\
\text { haemodialysis patients: } \\
\text { a systematic review and } \\
\text { meta-analysis. } 2011 \text {. }\end{array}$ & $\begin{array}{l}\text { Smart N., } \\
\text { Steele M. }\end{array}$ & $\begin{array}{l}\text { Revisión y } \\
\text { meta-aná- } \\
\text { lisis. }\end{array}$ & $\begin{array}{l}\text { Se realizó una búsqueda } \\
\text { sistemática de la litera- } \\
\text { tura en agosto del } 2010 \\
\text { para identificar ensayos } \\
\text { controlados y aleato- } \\
\text { rizados de estudios de } \\
\text { entrenamiento con ejer- } \\
\text { cicio en pacientes en } \\
\text { hemodiálisis. Posterior- } \\
\text { mente se realizó un me- } \\
\text { ta-análisis y la búsqueda }\end{array}$ & $\begin{array}{l}\text { Se seleccionaron ensayos contro- } \\
\text { lados aleatorios de entrenamiento } \\
\text { físico en pacientes en hemodiálisis. } \\
\text { Que incluyeran el estudio del V02, } \\
\text { calidad de vida, función cardíaca, } \\
\text { fuerza e ingesta de energía después } \\
\text { del entrenamiento físico }\end{array}$ & $\begin{array}{l}\text { Quince estudios, que rindie- } \\
\text { ron } 565 \text { pacientes fueron in- } \\
\text { cluidos, en donde el resultado } \\
\text { general fue que el entrena- } \\
\text { miento físico es seguro e im- } \\
\text { parte grandes mejoras en el } \\
\text { pico de V02 y la variabilidad } \\
\text { de la frecuencia cardíaca en } \\
\text { pacientes con ERC }\end{array}$ \\
\hline
\end{tabular}




\begin{tabular}{|c|c|c|c|}
\hline Título/Año & Autores & $\begin{array}{l}\text { Tipo } \\
\text { de estudio }\end{array}$ & Tamaño muestral \\
\hline & & & $\begin{array}{l}\text { se repitió en diciembre } \\
\text { de } 2010 \\
\text { El objetivo del estudio } \\
\text { fue cuantificar el cam- } \\
\text { bio inicial y observado } \\
\text { en el V02 pico, calidad } \\
\text { de vida, función cardía- } \\
\text { ca, fuerza y consumo } \\
\text { de energía después del } \\
\text { entrenamiento de ejer- } \\
\text { cicio en pacientes en } \\
\text { hemodiálisis }\end{array}$ \\
\hline
\end{tabular}

Evaluación de la calidad de vida relacionada con salud en pacientes en hemodiálisis periódica utilizando el KDQOL-SFTM. 2015

\section{Capote E.,} Argudí R., Mora S.

\section{Estudio descriptivo y de corte} transversal

Se trabajó con los 60 pacientes que cumplieron con los siguientes criterios: pacientes de 20 o más años; pacientes con tres o más meses de iniciado el tratamiento depurador; y que dieron su consentimiento para participar en el estudio

El objetivo del estudio fue evaluar la calidad de vida en pacientes en hemodiálisis

\section{Junqué A., Estudio Esteve V., prospectivo \\ Bernaveu $\mathrm{E}$.} Resultado de un
programa adaptado de ejercicio físico en pacientes ancianos en hemodiálisis. 2015

Estudio de 12 semanas de duración. 11 pacientes incluidos. Edad media 83.9 años y 37.2 meses en hemodiálisis
Preliminary study of an exercise programme for reducing fatigue and improving sleep among long-term haemodialysis patients. 2014
Con el objetivo de Analizar el efecto de un programa adaptado de ejercicio físico intradiálisis sobre la fuerza muscular, la capacidad funcional, la sintomatología depresiva y la calidad de vida en los pacientes ancianos ( $>75$ años) en hemodiálisis

Se aplicó a todos los pacientes el instrumento específico KDQOL-SFTM (Kidney Disease Quality of Life Short Form) versión 1.3 para valorar CVRS. Al cuestionario entregado a cada paciente, se adjuntó un documento de carácter explicativo, aunque cualquier tipo de duda se aclaró mediante la explicación verbal

Los datos que los pacientes no pudieron aportar se obtuvieron de sus familiares y/o de las historias clínicas ambulatorias del servicio de hemodiálisis

Los pacientes incluidos realizaron un programa de ejercicio físico adaptado mediante pelotas medicinales, pesas, bandas elásticas y cicloergómetros en las primeras dos horas de hemodiálisis. Se analizaron

\section{1.-Parámetros bioquímicos}

2.-Datos musculares: Fuerza extensión máxima de cuádriceps y hand-grip

3.-Test funcionales: "Sit to stand to sit" $y$ "six-minutes walking test"

4.-Sintomatología depresiva: Inventario Beck

\section{5.-Calidad de Vida: EuroQol-5D}

Al grupo de ejercicio se le realizó Maniam R., Estudio $\quad$ Se realizó un programa Subrama- quasi- expe- de ejercicios tres veces

nian $P_{\text {., }} \quad$ rimental por semana durante 12 Singh S. semanas antes de la HD a los pacientes de dos centros dialíticos. Los pacientes se clasificaron en el grupo de ejercicio $(n=28)$ o el grupo de control $(n=27)$
En este estudio, las dimensiones específicas con mayores puntuaciones fueron: relación con el personal de diálisis $(85,62)$, función cognitiva $(84,33)$ y calidad de la relación social $(82,88)$

Algunas dimensiones reportadas con puntuaciones altas, es decir, cercanas a 80 puntos, fueron: síntomas/problemas y soporte social. En este trabajo, esas dimensiones también tuvieron puntuaciones cercanas a 80 puntos, la primera con 77,03 y la segunda con 79,58 puntos

El programa adaptado de ejercicio físico intradiálisis mejoró la fuerza muscular, la capacidad funcional y la calidad de vida de nuestros pacientes ancianos en hemodiálisis

Aún en población anciana, nuestros resultados realzan los beneficios del ejercicio físico en los pacientes en hemodiálisis

Ante un paciente anciano en hemodiálisis, merece la pena considerar la realización de ejercicio físico adaptado intradiálisis como una parte más del cuidado integral en hemodiálisis

El ejercicio simple de intensidad baja a moderada es eficaz para mejorar la fatiga, los trastornos del sueño y la calidad de vida general entre los pacientes en hemodiálisis 


\begin{tabular}{|c|c|c|c|}
\hline Título/Año & Autores & $\begin{array}{l}\text { Tipo } \\
\text { de estudio }\end{array}$ & Tamaño muestral \\
\hline & & & $\begin{array}{l}\text { El objetivo fue determi- } \\
\text { nar la efectividad de un } \\
\text { programa de ejercicios } \\
\text { de baja a moderada in- } \\
\text { tensidad en prediálisis } \\
\text { para reducir la fatiga y } \\
\text { mejorar los trastornos } \\
\text { del sueño entre pacien- } \\
\text { tes de hemodiálisis }\end{array}$ \\
\hline
\end{tabular}

The Level of Anxiety and Depression in Dialysis Patients Undertaking Regular Physical Exercise Training -a Preliminary Study. 2016

\section{Dziubek W., Kowalska J., Kusztal M.}

Studio

clínico ex-

perimental

aleatorizado

Un total de 28 pacientes con insuficiencia renal etapa 5 (ESRD) completaron el estudio: 20 se asignaron al azar al entrenamiento aeróbico y 8 se asignaron al azar al entrenamiento de resistencia

El objetivo del estudio fue evaluar los efectos de un entrenamiento físico de seis meses realizado por pacientes en hemodiálisis (HD) sobre la depresión y la ansiedad

1. Anding K., Un ensayo

2. Bär T., 3. clínico de un TrojniakHennig J.

Incluyó a 46 pacientes con HD con un promedio de edad de 63.2 (24 hombres y 22 mujeres.
A structured exercise programme during haemodialysis for patients with chronic kidney disease: clinical benefit and long-term adherence. 2015 solo centro, no aleatorizado Que realizaron un programa de ejercicio (SPEP) durante 5 años. EI $78 \%$ de los pacientes completaron el programa después de 1 año y el $43 \%$ después de 5 años. Los participantes se dividieron -de acuerdo con el programa- en tres grupos: (1) grupo de alta adherencia ( $H A$, $>80 \%$ de 104 sesiones de entrenamiento en 12 meses), (2) adherencia moderada ( $M A$, $60-80 \%)$, y 3. Grupo de baja adherencia (LA, $<60 \%$ ))

Evidence of increased muscle atrophy and impaired quality of life parameters in patients with uremic restless legs syndrome. 2011
Giannaki C., Estudio Sakkas G., clínico Karatzaferi transversal
Usando los criterios del síndrome de piernas inquietas y la presencia de movimientos periódicos de las extremidades en sueño ( $R L S / h>15$ ),

\section{Actividad realizada}

narios autoinformados al inicio y después de la intervención. La percepción de los pacientes sobre el programa de ejercicio también se determinó mediante cuestionarios autoinformados se evaluaron 70 pacien-
La capacitación física se llevó a cabo al comienzo de las primeras 4 horas de diálisis, tres veces a la semana durante seis meses. En el estudio se utilizaron un cuestionario personal, el Inventario de Depresión de Beck (BDI) y el Inventario de Ansiedad Estado-Rasgo (STAI)

EI SPEP consistió en ejercicios dos veces a la semana durante 60 minutos durante la hemodiálisis, que incluía un entrenamiento combinado de resistencia (8 grupos musculares) y aeróbica (bicicleta ergométrica). La intensidad del ejercicio se ajustó continuamente a las mejoras de las pruebas de rendimiento. Los cambios en la resistencia y la capacidad de resistencia, el funcionamiento físico y la calidad de vida ( $Q \circ L)$ se analizaron durante 1 año, además de la adherencia a largo plazo y la economía del programa durante 5 años

La realización de entrenamiento físico durante la diálisis por pacientes con ESRD es beneficiosa para reducir sus niveles de ansiedad y depresión. Tanto el entrenamiento aeróbico como el de resistencia mejora el estado de ánimo, pero solo el entrenamiento de resistencia también resulta en la reducción de la ansiedad

El programa de ejercicios descrito mejora significativamente la función física y puede integrarse en una rutina de HD con una alta adherencia a largo plazo

El rendimiento físico se evaluó mediante una batería de pruebas: composición corporal mediante absorciometría de rayos $\mathrm{X}$ de energía dual, tamaño muscular y composición mediante tomografía computarizada, mientras que los síntomas
El bajo nivel de calidad de vida reportado por los pacientes con HD con síndrome de piernas inquietas parece deberse principalmente a la salud mental y aspectos relacionados con el sueño. También 


\begin{tabular}{|c|c|c|c|c|c|}
\hline Título/Año & Autores & $\begin{array}{l}\text { Tipo } \\
\text { de estudio }\end{array}$ & Tamaño muestral & Actividad realizada & Conclusiones principales \\
\hline & & & $\begin{array}{l}\text { tes de hemodiálisis clí- } \\
\text { nicamente estables y se } \\
\text { dividieron en los grupos } \\
\text { RLS ( } n=30 \text { ) y no RLS } \\
\text { ( } n=40) \\
\text { Los objetivos de la re- } \\
\text { visión fueron investigar } \\
\text { si los pacientes con HD } \\
\text { con RLS, en compara- } \\
\text { ción con aquellos sin el } \\
\text { síndrome, muestran evi- } \\
\text { dencia de una menor ca- } \\
\text { lidad de vida y del rendi- } \\
\text { miento físico general y } \\
\text { la capacidad funcional, } \\
\text { así como otras altera- } \\
\text { ciones en los índices de } \\
\text { composición corporal y } \\
\text { características muscula- } \\
\text { res, parámetros que po- } \\
\text { drían tener un impacto } \\
\text { significativo en la super- } \\
\text { vivencia y la calidad de } \\
\text { vida en esta población } \\
\text { de pacientes }\end{array}$ & $\begin{array}{l}\text { de depresión, percepción de calidad } \\
\text { del sueño y calidad de vida se eva- } \\
\text { luaron mediante cuestionarios vali- } \\
\text { dados }\end{array}$ & $\begin{array}{l}\text { se observa una mayor eviden- } \\
\text { cia de atrofia muscular en el } \\
\text { grupo de RLS y posiblemente } \\
\text { se puede atribuir a la falta de } \\
\text { sueño reparador }\end{array}$ \\
\hline $\begin{array}{l}\text { Single-blind randomi- } \\
\text { zed controlled trial to } \\
\text { evaluate the effect of } \\
6 \text { months of progres- } \\
\text { sive aerobic exercise } \\
\text { training in patients with } \\
\text { uraemic restless legs } \\
\text { síndrome. } 2010\end{array}$ & $\begin{array}{l}\text { Giannaki C., } \\
\text { Hadjigeor- } \\
\text { giou G., } \\
\text { Karatzaferi } \\
\text { C., Maridaki } \\
\text { M. Hadji- } \\
\text { georgiou G., } \\
\text { Karatzaferi } \\
\text { C., Maridaki } \\
\text { M. }\end{array}$ & $\begin{array}{l}\text { Estudio } \\
\text { clínico Ex- } \\
\text { perimental }\end{array}$ & $\begin{array}{l}24 \text { pacientes con síndro- } \\
\text { me de piernas inquietas } \\
(R L S) \text { en HD fueron } \\
\text { asignados aleatoriamen- } \\
\text { te a dos grupos: el gru- } \\
\text { po de entrenamiento } \\
\text { de ejercicio progresivo } \\
(n=12) \text { y el grupo de } \\
\text { ejercicio sin resistencia } \\
(n=12)\end{array}$ & $\begin{array}{l}\text { La sesión de ejercicio en ambos } \\
\text { grupos incluyó ciclismo intradiáli- } \\
\text { sis durante } 45 \text { minutos a } 50 \text { rpm. } \\
\text { Sin embargo, solo en el grupo de } \\
\text { entrenamiento progresivo se aplicó } \\
\text { resistencia, al } 60-65 \% \text { de la capa- } \\
\text { cidad máxima de ejercicio, que se } \\
\text { volvió a evaluar cada } 4 \text { semanas } \\
\text { para explicar la mejoría de los pa- } \\
\text { cientes. La severidad de los sínto- } \\
\text { mas del RLS se evaluó mediante la } \\
\text { escala de gravedad IRLSSG, la ca- } \\
\text { pacidad funcional por una batería } \\
\text { de pruebas, mientras que la calidad } \\
\text { del sueño, los niveles de depresión } \\
\text { y el estado de somnolencia diaria } \\
\text { se evaluaron mediante cuestiona- } \\
\text { rios validados, antes y después del } \\
\text { período de intervención }\end{array}$ & $\begin{array}{l}\text { Un programa de entrena- } \\
\text { miento de ejercicio progresi- } \\
\text { vo intradialítico de } 6 \text { meses } \\
\text { parece ser un enfoque seguro } \\
\text { y eficaz para reducir la gra- } \\
\text { vedad de los síntomas de RLS } \\
\text { en pacientes con HD. Parece } \\
\text { que las adaptaciones induci- } \\
\text { das por el ejercicio en todo } \\
\text { el cuerpo son en su mayoría } \\
\text { responsables de la reducción } \\
\text { en el puntaje de severidad } \\
\text { del RLS, ya que el ejercicio } \\
\text { sin protocolo de resistencia } \\
\text { aplicada no mejoró el estado } \\
\text { de gravedad del RLS de los } \\
\text { pacientes }\end{array}$ \\
\hline $\begin{array}{l}\text { Effect of acute and } \\
\text { chronic physical exer- } \\
\text { cise on patients with } \\
\text { periodic leg movements. } \\
2009\end{array}$ & $\begin{array}{l}\text { Maculano } \\
\text { A., Tu' Lio } \\
\text { De Mello M. }\end{array}$ & $\begin{array}{l}\text { Estudio } \\
\text { clínico Ex- } \\
\text { perimental }\end{array}$ & $\begin{array}{l}\text { Se seleccionaron } 22 \\
\text { voluntarios con patro- } \\
\text { nes de sueño alterados } \\
\text { diagnosticados por el } \\
\text { Sleep Intitute. Fueron } \\
\text { divididos en dos grupos } \\
\text { que se sometieron a } \\
\text { una prueba de esfuerzo } \\
\text { máximo y una polisom- } \\
\text { nografía (PSG) en la } \\
\text { misma noche } \\
\text { El objetivo del estudio } \\
\text { fue evaluar los efectos } \\
\text { del ejercicio intensivo } \\
\text { agudo y el ejercicio cró- }\end{array}$ & $\begin{array}{l}\text { El presente estudio se dividió en } \\
\text { dos experimentos: uno evaluó los } \\
\text { efectos del ejercicio físico intensivo } \\
\text { (prueba de esfuerzo máximo, mien- } \\
\text { tras que el otro analizó los efectos } \\
\text { del ejercicio físico crónico ( } 72 \text { se- } \\
\text { siones de entrenamiento aeróbico) } \\
\text { en voluntarios que presentaban } \\
\text { PLM } \\
\text { Se tomaron muestras de sangre de } \\
\text { grupos agudos y crónicos para la } \\
\text { dosificación de beta-endorfina }\end{array}$ & $\begin{array}{l}\text { El ejercicio físico puede me- } \\
\text { jorar los patrones de sueño y } \\
\text { reducir los niveles de PLM. } \\
\text { La correlación entre la libe- } \\
\text { ración de beta-endorfina des- } \\
\text { pués del ejercicio intensivo } \\
\text { agudo y los niveles de PLM } \\
\text { podría estar asociada con el } \\
\text { impacto que el ejercicio físi- } \\
\text { co tiene en el sistema opioi- } \\
\text { dérgico. Sugerimos que el } \\
\text { ejercicio físico puede ser un } \\
\text { tratamiento no farmacológico } \\
\text { útil para PLM }\end{array}$ \\
\hline
\end{tabular}




\begin{tabular}{|c|c|c|c|c|c|}
\hline Título/Año & Autores & $\begin{array}{l}\text { Tipo } \\
\text { de estudio }\end{array}$ & Tamaño muestral & Actividad realizada & Conclusiones principales \\
\hline & & & $\begin{array}{l}\text { nico en los patrones de } \\
\text { sueño en pacientes con } \\
\text { movimientos periódicos } \\
\text { de las piernas (PLM) }\end{array}$ & & \\
\hline $\begin{array}{l}\text { Interaction between In- } \\
\text { tradialytic Exercise and } \\
\text { Hemodialysis Adequacy. } \\
2013\end{array}$ & $\begin{array}{l}\text { Kirkman D., } \\
\text { Roberts L., } \\
\text { Kelm M. }\end{array}$ & $\begin{array}{l}\text { Ensayo } \\
\text { clínico } \\
\text { controlado }\end{array}$ & $\begin{array}{l}\text { Se reclutaron } 11 \text { pa- } \\
\text { cientes en hemodiáli- } \\
\text { sis }(H D) \text { (media de } 56 \\
\text { años) } \\
\text { El objetivo de este fue } \\
\text { probar si el ejercicio in- } \\
\text { tradialítico de suficiente } \\
\text { intensidad y duración } \\
\text { implementado en la se- } \\
\text { gunda mitad de la diáli- } \\
\text { sis puede ser tan eficaz } \\
\text { como aumentar el tiem- } \\
\text { po de diálisis para la } \\
\text { adecuación de la diálisis }\end{array}$ & $\begin{array}{l}\text { Cada paciente completó tres eta- } \\
\text { pas de prueba en orden aleatorio: } \\
\text { cuidado de rutina (CONT), aumen- } \\
\text { to del tiempo de HD de } 30 \text { min } \\
\text { (TIEMPO) y ejercicio intradiáli- } \\
\text { sis (EXER), } 60 \text { min de ciclismo al } \\
90 \% \text { del umbral de lactato en los } \\
\text { últimos } 90 \text { min de HD }\end{array}$ & $\begin{array}{l}\text { El ejercicio intradialítico ha- } \\
\text { cia el final de la HD no puede } \\
\text { reemplazar la prescripción } \\
\text { de un aumento del tiempo de } \\
\text { HD para la adecuación de la } \\
\text { diálisis, pero puede ser una } \\
\text { terapia complementaria para } \\
\text { el control del fosfato sérico }\end{array}$ \\
\hline $\begin{array}{l}\text { Effect of exercise } \\
\text { training on chronic } \\
\text { inflammation. } 2010\end{array}$ & $\begin{array}{l}\text { Beavers K., } \\
\text { Brinkley T., } \\
\text { Nicklas B. }\end{array}$ & $\begin{array}{l}\text { Revisión } \\
\text { sistemática }\end{array}$ & $\begin{array}{l}\text { Esta revisión incluyó } 26 \\
\text { estudios observaciona- } \\
\text { les que examinan la aso- } \\
\text { ciación entre los marca- } \\
\text { dores de inflamación } \\
\text { sistémica y la actividad } \\
\text { física autoinformada o } \\
\text { la capacidad aeróbica } \\
\text { medida directamente } \\
\text { En este estudio solo se } \\
\text { incluyeron ensayos con- } \\
\text { trolados aleatorios pu- } \\
\text { blicados hasta la fecha } \\
\text { de su investigación }\end{array}$ & $\begin{array}{l}\text { Resume la evidencia a favor y en } \\
\text { contra del papel del aumento de la } \\
\text { actividad física en la reducción de } \\
\text { la inflamación crónica. Los grandes } \\
\text { estudios de cohorte basados en la } \\
\text { población muestran consistente- } \\
\text { mente una asociación inversa en- } \\
\text { tre los marcadores de inflamación } \\
\text { sistémica y la actividad física o el } \\
\text { estado físico. Los datos de varios } \\
\text { estudios de intervención a pequeña } \\
\text { escala respaldan que el entrena- } \\
\text { miento físico disminuye la inflama- } \\
\text { ción }\end{array}$ & $\begin{array}{l}\text { Hay poca evidencia de tera- } \\
\text { pias que puedan tratar efi- } \\
\text { cazmente a individuos con } \\
\text { marcadores elevados de in- } \\
\text { flamación que se encuentran } \\
\text { dentro del rango clínicamente } \\
\text { normal. Sin embargo, datos } \\
\text { consistentes de estudios ob- } \\
\text { servacionales que muestran } \\
\text { un vínculo entre los niveles } \\
\text { autoinformados de actividad } \\
\text { física y biomarcadores infla- } \\
\text { matorios, así como algunos } \\
\text { datos positivos prometedores } \\
\text { de ensayos controlados alea- } \\
\text { torios, indican que el aumento } \\
\text { de la actividad física aeróbica } \\
\text { podría ser eficaz para reducir } \\
\text { la inflamación crónica, espe- } \\
\text { cialmente en individuos con } \\
\text { enfermedades crónicas aso- } \\
\text { ciadas con un estado de infla- } \\
\text { mación elevada }\end{array}$ \\
\hline
\end{tabular}

Se describen los efectos del ejercicio en hemodiálisis en el sistema cardiovascular, en el metabolismo mineral óseo, en la función y capacidad física, en la calidad de vida con los beneficios psicológicos y en la eliminación de solutos de los pacientes en hemodiálisis.

\section{Efectos Cardiovasculares}

Las enfermedades cardiovasculares (ECV) son la primera causa de muerte en los pacientes con ERC ter- minal en tratamiento dialítico. A medida que progresa la ERC también aumenta el riesgo de morbi-mortalidad cardiovascular en estos pacientes. Se estima una mortalidad de $56,4 \%$ en pacientes que requieren de HD crónica, de los cuales, más de la mitad mueren por ECV 5,6 . Estas enfermedades cardiovasculares son causadas por la calcificación vascular y el aumento de marcadores inflamatorios y protrombóticos, lo que lleva a que se produzcan alteraciones en la capa íntima (disfunción endotelial) y capa media (rigidez arterial) de la pared de los vasos sanguíneos ${ }^{6,7}$. 
Los datos clínicos muestran que la disfunción endotelial ocurre tempranamente en el curso de la insuficiencia renal y predispone la aterosclerosis acelerada en pacientes con ERC, que se traduce clínicamente en un resultado Cardiovascular deficiente ${ }^{8}$. Por otro lado, el daño de la capa media, que típicamente involucra calcificación, altera la elasticidad arterial e induce rigidez arterial. Las manifestaciones clínicas de rigidez arterial incluyen hipertensión sistólica, aumento de la sobrecarga miocárdica con hipertrofia ventricular izquierda, disminución de la perfusión subendocárdica, disfunción diastólica y, eventualmente, insuficiencia cardíaca?.

Existe reciprocidad entre la disfunción endotelial y la rigidez arterial. Al exponer las células endoteliales a un mayor estrés biomecánico, la rigidez arterial puede agravar la disfunción endotelial, mientras que, en la disfunción endotelial, la alteración de la biodisponibilidad del factor de relajación óxido nítrico (ON) aumenta la rigidez arterial ${ }^{8}$.

El ejercicio físico posee efectos beneficiosos bien conocidos en el corazón, el músculo esquelético y pared vascular. Además de la tradicional modificación de los factores de riesgo, el ejercicio mejora la salud vascular a través del aumento de la biodisponibilidad de $0 \mathrm{~N}$, efectos antioxidantes y antiinflamatorios generalizados. Como tal, la actividad física regular se recomienda insistentemente en Europa ${ }^{10}$. En pacientes en hemodiálisis, se sugirió que un programa de ejercicios aeróbicos de dos veces por semana durante 3 meses mejora significativamente el índice de aumento aórtico (AIx) y la velocidad de la onda de pulso aórtica (ambos marcadores de rigidez arterial) que volvieron a los niveles basales en el lapso de un mes después del desentrenamiento ${ }^{11}$.

En un estudio donde un grupo de pacientes realizó 40 minutos de ejercicios de fuerza y resistencia de intensidad algo extenuante durante 3 meses, se logró una significativa reducción de la rigidez arterial y un aumento significativo sobre la masa magra, sin variaciones importantes en el resto de ítems de composición corporal $^{12}$. Con respecto a la reparación endotelial, el ejercicio físico con un entrenamiento aeróbico de intensidad moderada durante 6 meses también ha sugerido un aumento del reclutamiento de células progenitoras endoteliales (CPE) que se ha relacionado con la mejora de la función endotelial en varias poblaciones, incluyendo los pacientes con $\mathrm{ERC}^{13}$.
El ejercicio también ha indicado ser eficaz en la reducción de la presión arterial (PA) sistólica y diastólica, que representa un alto riesgo cardiovascular en sujetos con ERC. Un meta-análisis reveló que la combinación del entrenamiento aeróbico y de resistencia, y el entrenamiento aeróbico de alta intensidad tienen un efecto superior en la reducción de la $\mathrm{PA}^{14}$.

La evidencia sobre la fisiopatología de la enfermedad vascular en la ERC y los posibles efectos vasculoprotectores del ejercicio revelan efectos clínicamente beneficiosos para la reducción el riesgo cardiovascular en estos pacientes. Es por esto por lo que las personas con ERC deben ser aconsejados y regularmente alentados por el personal de nefrología para aumentar su nivel de actividad física. Es recomendable incentivar el ejercicio físico en una intensidad moderada durante $30 \mathrm{mi}$ nutos en la mayoría de los días de la semana, prestando atención al seguimiento regular ${ }^{15}$.

\section{Efectos en el metabolismo mineral óseo}

Las anomalías óseas son una causa importante de morbilidad y disminución de la calidad de vida de los pacientes con ERC ${ }^{16}$.

La enfermedad ósea de los pacientes en HD se debe principalmente al efecto del hiperparatiroidismo secundario (HPT). Los pacientes en diálisis desarrollan resistencia ósea a la acción de la PTH por lo que necesitan niveles mayores para conseguir el remodelado óseo normal. Si presentan niveles crónicamente elevados de PTH desarrollan osteítis fibrosa (enfermedad ósea de alto remodelado) y, por el contrario, si los niveles de PTH se mantienen normales se asocia con hueso adinámico (enfermedad ósea de bajo remodelado $)^{17}$.

El estrés repetitivo aplicado a sitios que soportan peso durante largos periodos de tiempo refuerza los huesos, por lo que, tanto los ejercicios de resistencia de alta y baja intensidad pueden cambiar los índices bioquímicos de recambio óseo. Además, el entrenamiento de fuerza puede aumentar de manera regional la densidad mineral ósea (DMO) aumentando la formación ósea. Los reguladores endocrinos del hueso, como la $\mathrm{PTH}$, la vitamina $D$ y el calcio, también pueden verse afectados durante los períodos de ejercicio extenuante y pueden mejorar el metabolismo óseo. Un estudio realizado por Marinho y cols. sugiere que un programa de ejercicios de resistencia de 24 semanas intradiálisis es eficaz para mejorar la DM0, especialmente en el cuello femo- 
ral junto con una leve reducción de los niveles de PTH (clínicamente significativo) $^{16}$.

\section{Efectos en la función y capacidad física}

Los pacientes con ERC que reciben tratamiento de hemodiálisis tienen una considerable disminución en la tolerancia al ejercicio, en la capacidad funcional, en la resistencia y en la fuerza. Además, sufren una mayor pérdida de masa muscular que, junto con la anemia, son factores claves en la disminución de la capacidad funcional $^{18}$.

Al revisar la literatura nos encontramos con que la ejecución de ejercicio aeróbico durante la HD mejora la capacidad funcional, proporcionando beneficios cardiorrespiratorias y musculares a los pacientes. Los estudios han propuesto que el ejercicio promueve la mejora de la capacidad aeróbica y fitness, disminución de la fatiga y la ansiedad, mejora la capilaridad muscular y presión arterial en reposo ${ }^{19}$. Un estudio realizado por Groussard y cols. ejecutó un programa de entrenamiento aeróbico intradialítico de 3 meses, (ciclismo aerobio) 3 veces por semana durante 30 min cada sesión. La aptitud física se evaluó mediante una prueba de laboratorio objetiva, es decir, V02 máximo, y por una prueba de campo que mide el rendimiento físico de una tarea estandarizada, es decir, tm6m (test de marcha 6 minutos). Aunque el entrenamiento aeróbico no tuvo ningún efecto sobre V02 máximo, mejoró la distancia recorrida $(+23,4 \%)$. Esta discrepancia se puede deber a que los cambios inducidos por el entrenamiento en V02 máximo están positivamente correlacionados con la duración del entrenamiento de ejercicio ${ }^{20}$. Según Smart y Steele, los cambios más importantes se han observado en pacientes que realizan entrenamiento aeróbico combinado y entrenamiento de fuerza durante 60 más meses en días sin diálisis ${ }^{21}$.

En otro estudio, un total de 13 pacientes fueron sometidos a un programa de ejercicios de 2 sesiones semanales, 1 hora previo a la hemodiálisis, durante 8 semanas. El entrenamiento consistía en ejercicios calisténicos de grupos musculares del tren inferior. La intervención logró cambios significativos respecto a la fuerza muscular, metros recorridos en el test de marcha de 6 minutos y disminución en la presión arterial diastólica².

\section{Efectos psicológicos y en la calidad de vida}

La calidad de vida relacionada con la salud (CVRS) es un concepto de aparición más o menos reciente y se identifica como el conjunto de factores relacionados con el estado de salud que forman parte del concepto multidimensional de calidad de vida, tal y como lo definió la OMS: "la percepción personal de un individuo de su situación en la vida, dentro del contexto cultural y de valores en que vive y en relación con sus objetivos, expectativas, valores e intereses" ${ }^{\prime \prime 22}$.

En un estudio realizado por Junqué Jiménez y cols., la introducción de un programa de ejercicio físico adaptado (aeróbico) en pacientes ancianos ( $>75$ años) que requieren HD mejoró la fuerza muscular, la capacidad funcional, la sintomatología depresiva y la calidad de vida.

En relación a la sintomatología depresiva, los resultados refuerzan los beneficios del ejercicio descritos en estudios anteriores. Su explicación se basa en aspectos fisiológicos como la liberación de ciertos neurotransmisores como las endorfinas al torrente circulatorio provocando una sensación completa de bienestar; y, por otro lado, en diversos aspectos emocionales y conductuales como la sustitución de pensamientos negativos y baja autoestima, disminución de la ansiedad y mejoría notable del humor así como un incremento de las relaciones sociales al realizar una actividad divertida, dirigida y programada en el transcurso de las sesiones de HD $23,24,25$.

Otro estudio, realizado por Radha Maniam y Pathmawathi Subramanian, reveló que un programa de ejercicios de flexibilidad y fortalecimiento, a un rango de esfuerzo máximo del $60 \%$ al $80 \%$, podría mejorar la fatiga y la calidad del sueño en los pacientes de hemodiálisis a largo plazo. Luego de que los pacientes realizaran este programa, varios informaron que estaban mejor capacitados para realizar tareas de rutina y que se sentían más energéticos después del tratamiento. Del mismo modo, también indicaron un mejor sueño nocturno después del tratamiento, probablemente por sentirse menos fatigados durante el día y no haber tenido la necesidad de un sueño diurno. Estos cambios trascendieron considerablemente en la calidad de vida, donde se vio una mejoría general ${ }^{26}$. Por otro lado, Dziubek evaluó el efecto de 6 meses de ejercicio aeróbico y de fuerza muscular, en pacientes en hemodiálisis, sobre los síntomas de depresión y ansiedad. Realizó un entrenamiento de 3 veces por semana durante las primeras dos horas de hemodiálisis, donde se concluyó que existen disminuciones significativas respecto a los síntomas de depresión y solo en un ítem en los síntomas de ansiedad ${ }^{27}$.

Anding, en el 2015 realizó un estudio en el cual sugiere que un entrenamiento combinado de fuerza y resistencia, con ajustes constantes de intensidad, dos veces por 
semana durante 60 minutos, mejora significativamente la calidad de vida en las subescalas de función física, desempeño físico y limitaciones emocionales, evaluadas mediante el cuestionario SF36 ${ }^{28}$.

El síndrome de piernas inquietas (RLS por su nombre en inglés) de tipo urémico, ha atraído la atención creciente durante la última década desde que la evidencia publicada reveló que esta forma de RLS secundaria podría inducir en la reducción de la calidad de vida del paciente, la depresión y la calidad del sueño, mientras que la gravedad de los síntomas se asoció con un aumento en el riesgo de muerte. Además, estudios recientes refieren una posible asociación del RLS urémico con la atrofia muscular y contribución a los movimientos periódicos de las extremidades en el sueño, una característica común de los pacientes RLS ${ }^{29}$.

Un programa de entrenamiento de ejercicio aeróbico de resistencia progresiva intradiálisis de 14 semanas redujo la gravedad de los síntomas de RLS en un $42 \%$ en comparación con un grupo de control que no ejercitaba. En ese estudio, también se observaron mejoras significativas en los niveles de calidad de vida, la calidad del sueño y la puntuación de la depresión en el ejercicio de los pacientes con $\mathrm{HD}^{30}$. Los mecanismos por el cual el entrenamiento progresivo de ejercicio puede reducir la sintomatología del RLS todavía no están claro, sin embargo, en un estudio de pacientes con RLS no urémico, se observó una relación inversa entre la liberación de ß-endorfinas después del entrenamiento físico y el índice de movimientos periódicos de las extremidades $^{31}$. La mejora de los síntomas del RLS a través de un aumento de los niveles de opioides, como lo son las B-endorfinas, parece ser uno de los candidatos más fuertes como mecanismo, ya que se sabe que el RLS está relacionado con un sistema opioide defectuoso en el cerebro en este tipo de pacientes ${ }^{32}$.

\section{Efectos en la eliminación de solutos}

Las estrategias terapéuticas para mejorar la idoneidad de la diálisis, incluida la eliminación de solutos distintos de la urea, están altamente justificadas. Una estrategia sugerida es ejercitarse durante la diálisis. El modelado de la ecuación que simula el ejercicio intradialítico predice que la hiperemia a los tejidos de baja perfusión como el músculo aumentará el aclaramiento de urea. Se ha descrito que el aumento de la idoneidad de la diálisis con 60 min de ejercicio intradialítico es equivalente al aumento del tiempo de diálisis en $20 \mathrm{mi}-$ nutos, sin embargo, el ejercicio intradialítico no puede reemplazar la prescripción del tiempo de hemodiálisis, pero si puede ser una terapia complementaria para el control del fósforo sérico ${ }^{33}$.

Es importante destacar que la mayoría de la depuración de urea se produce durante la primera mitad de la diálisis. Si se realiza ejercicio durante la segunda mitad de la diálisis cuando las concentraciones plasmáticas de urea más bajas son evidentes, entonces se establecerían mayores gradientes de concentración entre el tejido de baja perfusión y el plasma. Además, el ejercicio regular moderado puede modular la respuesta inflamatoria, lo que sería beneficioso en ERC terminal34,35. Esto daría como resultado un mayor flujo difusivo en la urea del tejido al plasma, maximizando la eficacia del ejercicio para la adecuación de la diálisis. En apoyo, se ha pronosticado matemáticamente que, mediante el mantenimiento de un flujo sanguíneo alto a los tejidos de baja perfusión en la última parte de la diálisis, la post-diálisis de recuperación de urea se eliminará33.

\section{Implicaciones para la práctica clínica}

De acuerdo con lo descrito, se recomienda la implementación o indicación de ejercicios aeróbicos tanto predialiticos como intradialiticos, con una frecuencia de 2 a 3 veces por semana y una duración de 30 minutos, ya que estos podrían mejorar la rigidez vascular, la presión arterial, la capacidad aeróbica, el metabolismo óseo y eliminación de solutos, lo que se puede considerar como terapia complementaria a la hemodiálisis crónica.

\section{Conclusiones}

En este estudio se evidencia que el ejercicio físico tiene una gran cantidad de beneficios para los pacientes con ERC que se encuentran en tratamiento dialítico. Sin embargo, los programas de ejercicios para estos pacientes no se utilizan como terapia complementaria, pese a ser una forma económica de ayudar a minimizar problemas asociados a dicha enfermedad, como lo son las ECV, alteraciones en la capacidad y función física, disminución de la calidad de vida, entre otros.

Incluir programas de ejercicios intradialíticos es una buena forma de ayudar, no solo en el área fisiológica del paciente, sino que también en el área psicológica y social ya que al realizar actividades grupales incrementan las relaciones sociales y mejora la calidad de vida, disminuyendo síntomas de depresión. 


\section{El autor declara que no hay conflicto de interés.}

\author{
Recibido: 5 febrero 2017 \\ Revisado: 4 agosto 2017 \\ Modificado: 12 noviembre 2017 \\ Aceptado: 4 mayo 2018
}

\section{Bibliografía}

1. Poblete Badal H. Registro de Diálisis. Sociedad Chilena de Nefrología. XXXV Cuenta de hemodiálisis crónica (HDC) en Chile. [Internet]. 31 Ago 2015 [Consultado 17 Nov 2017] Disponible en: https:// www.nefro.cl/v2/biblio/registro/21.pdf

2. Cigarroa I, Barriga R, Michéas C, Zapata-Lamana R, Soto C, Manukian T. Efectos de un programa de ejercicio de fuerza-resistencia muscular en la capacidad funcional, fuerza y calidad de vida de adultos con enfermedad renal crónica en hemodiálisis. Rev Med Chile 2016;144:844-52.

3. Greenwood SA, Naish P, Clark R, O'Connor E, Pursey VA, Macdougall IC et al. Intra-dialytic exercise training: a pragmatic approach. J Ren Care 2014; 40(3):219-26.

4. Segura-Ortí E. Ejercicio en pacientes en hemodiálisis: revisión sistemática de la literatura. Nefrologia 2010;30(2):236-46.

5. Ventura J. Riesgo cardiovascular en pacientes con enfermedad renal crónica. Rev Urug Cardiol 2006;21(2):143-57.

6. Van Craenenbroeck A, Van Craenenbroeck E, Kouidi $E$, Vrints C. Vascular effects of exercise training in ckd: current evidence and pathophysiological mechanisms. Clin J Am Soc Nephrol. 2014; 9(7):1305-18.

7. González I, Casanova C, Escobar C, García A, Peraira J. Enfermedad cardiovascular y función renal. Mecanismos patogénicos. Rev Esp Cardiol Supl. 2008;8(E):10-21

8. Briet M, Burns KD. Chronic kidney disease and vascular re-modelling: Molecular mechanisms and clinical implications. Clin Sci (Lond) 2012;123(7):399-416.
9. Orozco R. Enfermedad cardiovascular (ECV) en la enfermedad renal crónica (ERC). Rev Med Clin Condes 2015; 26(2):142-55.

10. Schuler G, Adams V, Goto Y. Role of exercise in the prevention of cardiovascular disease: results, mechanisms, and new perspectives. Eur Heart $\mathrm{J}$ 2013;34(24):1790-9.

11. Mustata S, Chan C, Lai V, Miller JA. Impact of an exercise program on arterial stiffness and insulin resistance in hemodialysis patients. J Am Soc Nephrol 2004;15(10):2713-8.

12. Mihaescu A, Avram C, Bob F, Gaita D, Schiller 0, SchiIler A. Benefits of exercise training during hemodialysis sessions: a prospective cohort study. Nephron Clin Pract. 2013;124(1-2):72-8.

13. Manfredini F, Rigolin GM, Malagoni AM, Soffritti S, Boari B, Conconi et al. Exercise capacity and circulating endothelial progenitor cells in hemodialysis patients. Int $\mathrm{J}$ Sports Med 2007;28(5): 368-73.

14. Heiwe S, Jacobson SH. Exercise training for adults with chronic kidney disease. Cochrane Database Syst Rev. 2011 0ct 5;(10):CD003236. doi: 10.1002/14651858.CD003236.pub2.

15. Roshanravan B, Robinson-Cohen C, Patel KV, Ayers $E$, Littman AJ et al. Association between physical performance and all cause mortality in CKD. J Am Soc Nephrol 2013;24(5):822-30.

16. Marinho SM, Moraes C, Barboza JESM, Carraro Eduardo JC, Fouque D et al. Exercise training alters the bone mineral density of hemodialysis patients. J Strength Cond Res. 2016;30(10):291823.

17. Coyne DW, Cheng SC, Delmez JA. Capítulo 35: Enfermedad ósea. En: Daugirdas JT. Manual de diálisis. $4^{a}$ Edición. Barcelona: Lippincott Williams \& Wilkins; 2008. p. 572.

18. Hernández Sánchez $S$, García López $D$, Santos Lozano A, González-Calvo G, Brazález Tejerina M, Garatachea Vallejo N. Valoración física, condición física y calidad de vida en pacientes con diferentes tratamientos renales sustitutivos. Enferm Nefrol 2015; 18(2):81-8. 
19. Böhm J, Borba M, Saldanha F. Efeitos do exercício aeróbio durante a hemodiálise em pacientes com doença renal crônica: uma revisão da literatura. J Bras Nefrol 2012;34(2):189-194.

20. Groussard C, Rouchon-Isnard M, Coutard C, Romain $F$, Malardé L, Lemoine-Morel $S$ et al. Beneficial effects of an intradialytic cycling training program in patients with end-stage kidney disease. Appl Physiol Nutr Metab. 2015;40(6):550-6.

21. Smart N, Steele M. Exercise training in haemodialysis patients: a systematic review and meta-analysis. Nephrology (Carlton). 2011;16(7):626-32.

22. Capote E. Evaluación de la calidad de vida relacionada con salud en pacientes en hemodiálisis periódica utilizando el KDQOL-SFTM. Medisur 2015;13(4): 508-16.

23. Junqué Jiménez $A$, Esteve Simó $V$, Tomás Bernaveu $E$, Paz López 0, Iza Pinedo G, Luceño Solé I et al. Resultado de un programa adaptado de ejercicio físico en pacientes ancianos en hemodiálisis. Enferm Nefrol 2015;18(1):11-8.

24. Kouidi E, Iacovides A, Iordanidis $P$, Vassiliou $S$, Deligiannis $A$, Ierodiakonou $C$, Tourkantonis $A$. Exercise renal rehabilitation program: psychosocial effects. Nephron. 1997;77(2):152-8.

25. Suh MR, Jung HH, Kim SB, Park JS, Yang WS. Effects of regular exercise on anxiety, depression, and quality of life in maintenance hemodialysis patients. Ren Fail. 2002;24(3):337-45.

26. Maniam R, Subramanian P. Preliminary study of an exercise programme for reducing fatigue and improving sleep among long-term haemodialysis patients. Singapore Med J 2014;55(9):476-82.

27. Dziubek W, Kowalska J, Mariusz Kusztal. The Level of Anxiety and Depression in Dialysis Patients Undertaking Regular Physical Exercise Training - a Prelimi- nary Study. Kidney Blood Press Res. 2016;41(1):8698.

28. Anding K, Bär T, Trojniak-Hennig J, Kuchinke S, Krause $\mathrm{R}$ et al. A structured exercise programme during haemodialysis for patients with chronic kidney disease: clinical benefit and long-term adherence. BMJ Open. 2015;27;5(8):e008709.

29. Giannaki CD, Sakkas GK, Karatzaferi C, Hadjigeorgiou GM, Lavdas E, Liakopoulos V et al. Evidence of increased muscle atrophy and impaired quality of life parameters in patients with uremic restless legs syndrome. PLoS One. 2011;6(10):e25180.

30. Giannaki CD, Hadjigeorgiou GM, Karatzaferi C, Maridaki MD, Koutedakis $Y$, Founta $P$ et al. A single-blind randomized controlled trial to evaluate the effect of 6 months of progressive aerobic exercise training in patients with uraemic restless legs syndrome. Nephrol Dial Transplant. 2013 Nov;28(11):2834-40.

31. Esteves AM, de Mello MT, Pradella-Hallinan M, Tufik S. Effect of acute and chronic physical exercise on patients with periodic leg movements. Med Sci Sports Exerc. 2009;41(1):237-42.

32. von Spiczak S, Whone AL, Hammers A, Asselin MC, Turkheimer $F$, Tings $T$ et al. The role of opioids in restless legs syndrome: an $[11 \mathrm{C}]$ diprenorphine PET study. Brain. 2005;128(Pt 4):906-17.

33. Kirkman DL, Roberts LD, Kelm M, Wagner J, Jibani $\mathrm{MM}$, Macdonald $\mathrm{JH}$. Interaction between intradialytic exercise and hemodialysis adequacy. Am J Nephrol. 2013;38(6):475-82.

34. Leypoldt JK. Kinetics of B 2 -microglobulin and phosphate during hemodialysis: effects of treatment frequency and duration. Semin Dial. 2005;18(5):401-8.

35. Beavers KM, Brinkley TE, Nicklas BJ. Effect of exercise training on chronic infl ammation. Clin Chim Acta 2010; 411(11-12):785-93.

Este artículo se distribuye bajo una Licencia Creative Commons Atribución-NoComercial 4.0 Internacional. https://creativecommons.org/licenses/by-nc/4.0/

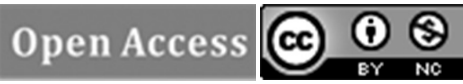

